\title{
The numbers games
}

\author{
Øystein E. Olsen ${ }^{1}$
}

Received: 28 October 2015 / Accepted: 12 November 2015 / Published online: 21 December 2015

(C) Springer-Verlag Berlin Heidelberg 2015

In 1979 Allan M. Cormack and Godfrey N. Hounsfield were awarded the Nobel Prize in medicine "for the development of computer assisted tomography". By this time, Hounsfield had published eight papers in medical journals, his seminal works in the British Journal of Radiology. The second and, thus far, last Nobel Prize awarded for medical imaging went to Paul C. Lauterbur and Peter Mansfield, belatedly, in 2003 "for their discoveries concerning magnetic resonance imaging". Fourteen of Mansfield's works, including his most important, had also appeared in the British Journal of Radiology. (He published twice in Pediatric Radiology [1, 2]). This year medical journals are expected to publish almost six times the number of radiologyrelated articles compared with the Nobel year 1979 and about twice as many as in 2003 (Fig. 1). At least part of this publishing explosion must be explained by increased scientific activity. Witnessing such an unprecedented increase in productivity begs two questions: Where is the big discovery that will launch the next transformation of medical imaging? Or is there perhaps no direct link between article output and scientific impact?

In the domain of journal publishing, the Impact Factor has been around for decades. Everyone agrees "it is just another number" and "we would never obey its dictate". But try securing a research grant if you have not appeared in highimpact journals. Or try to publish something unconventional, controversial or counter-current (innovative?) in the most prestigious journals. Our money would be on rather disap-

Øystein E. Olsen

oeolsen.pedrad@me.com

1 Radiology Department,

Great Ormond Street Hospital for Children NHS Foundation Trust, Great Ormond Street,

London WC1N 3JH, UK pointing outcomes. This is speculation and a virtual kicking of our own backsides, but it is at least an interesting paradox that the most important works on which two Nobel Prizes were founded were published in the fringes of the medical literature (the British Journal of Radiology, host to the laureates, had a 1992 impact factor of less than 0.7). Journal editors claim not to be blinded by numerics, but which editor is happy to see her journal slide down the ranking? A self-perpetuating relationship emerges: Authors need to publish high volumes and in high-ranking journals; journals favour works that are guaranteed citations. The mechanics of the phenomenon are fairly straight-forward: It is much easier to rank by numbers than by any qualitative dimension. The unfortunate consequence may be a profession that self-restricts its research to topics that are conventional, non-controversial and therefore guaranteed publication.

In 2014 Pediatric Radiology received 850 new article submissions, about one-fifth the total number of radiology articles published in all medical journals in 1979. The drive to publish is clearly increasing, and there must be several underlying forces. One obviously is technology: It is easier than ever to collect clinical and imaging data, transform these into a manuscript and submit-to-journal by the tap of an index finger. That this is phenomenal can be boded for by anyone who published in science before the internet era, in times when a whole support apparatus had to be enrolled to clear all the required hurdles. Now, everyone can publish. With the internet came an astonishing democratisation of medical publishing (ease of article submission, and also the birth of the onlineonly journal), which is fundamentally sound. A second driving force takes the shape of employer and research-funding organisations, and in particular their strategies for selecting whom to employ, support and award. One way is by counting and weighting (by journal-ranking) publications; both fair and objective. With modern metrics it is no longer a question of 
Fig. 1 Plot of PubMed entries 1970-2013 for journal articles with a radiology topic (paediatric separately in red) shows a nearexponential increase. Soon, the majority of papers will have been published subsequent to the hitherto only two Nobel Prizes for medicine awarded in radiology.

Can we expect another significant discovery soon, or is there perhaps no notable relation between publication quantity and clinical impact?

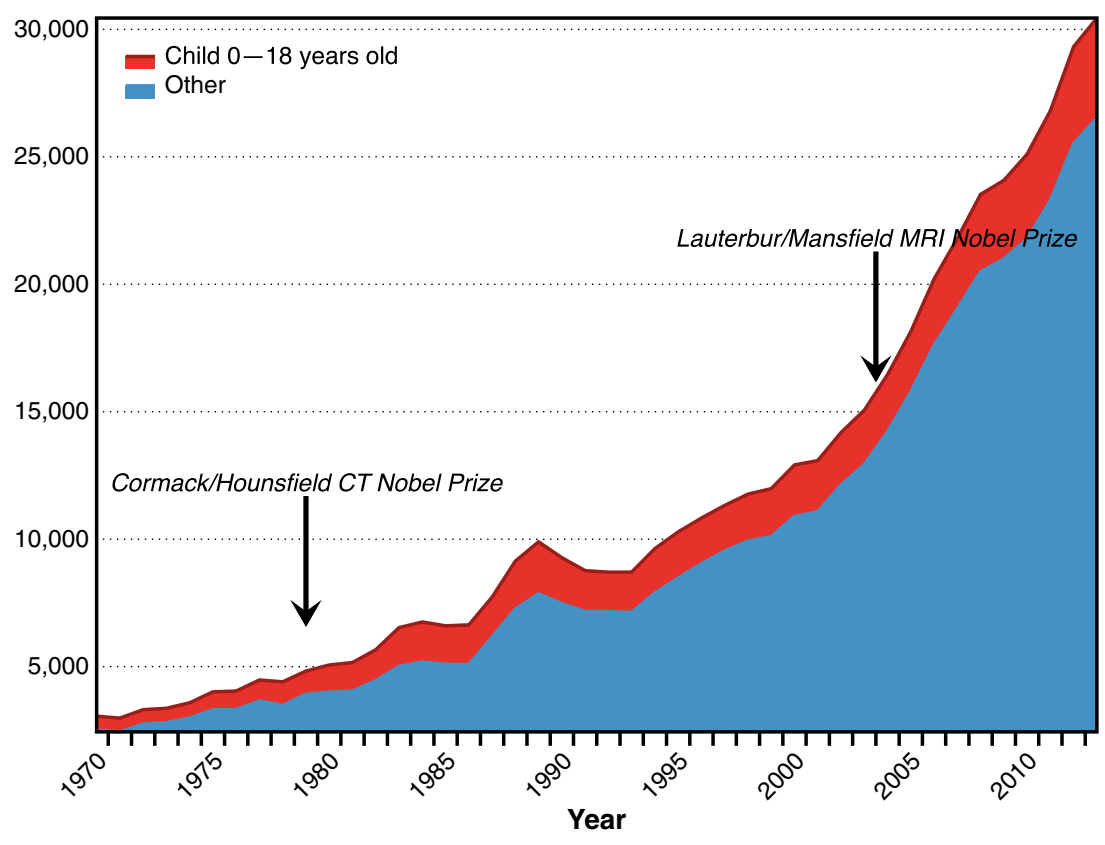

how capable you are or which ideas you have - now, exact and unsentimental ranking rules.

But is such quantification merely a route of least resistance in a world that is increasingly complex?

Has the body of attempted and de facto publications become so bloated that most of its signal has disappeared in the noise of obviousness and regurgitant? Luckily we are obliged only to post the question, and so can grant the reader the delight of attempting an answer. Let us only set the scene by relating the question to some of the greatest problems (politically correct: challenges; even more politically correct: opportunities) our profession is faced with now: de-professionalisation, commoditisation and commercialisation. Attempts at taming the powerful professions are as old as politics itself. Different times see this process in different guises. In our time its prevailing incarnation is bean-counting. Politicians and managers (both groups with shorter sell-by dates than professionals) increasingly govern by defining targets for their organisations. It is no surprise to find that these targets are commonly simple metrics (numbers of reports and report turn-around time, for example) rather than something that actually matters (added value in clinical care, for example). The more we as a profession engage with the idea of simplistic "production targets" the more we surrender quality for quantity. The radiologist may attain the required number of reports within the target timeframe but on the way may have faded to invisible, both to patients and clinical colleagues. Clinically added value is lost in busy-ness, and up next is replacement by (cheaper) remote labour, if not by a computer [3].

As scientific journal editors we are given two important tasks: identify what is hampering scientific development, and attempt to facilitate counteraction. Creation of Nobel laureates is not mandatory so long as we contribute to regaining the profession's power to define its own development (as opposed to the bending forces of politically imposed regulation or indeed of whatever manufacturers find commercially viable at the moment). Our assignment is to encourage critical appraisal of imaging equipment, clinical practice and engrained ideas. There are many questions still to be answered and an untold number still to be asked. There are many concepts to be translated from drawing board to clinic by means of rigorously probing and improving their efficacies. For this, numbers will certainly help, but numbers remain simple in a world that is increasingly more involved.

\section{Compliance with ethical standards}

Conflicts of interest None

\section{References}

1. Chrispin A, Small P, Rutter N et al (1986) Transectional echo planar imaging of the heart in cyanotic congenital heart disease. Pediatr Radiol 16:293-297

2. Chrispin A, Small P, Rutter N et al (1986) Echo planar imaging of normal and abnormal connections of the heart and great arteries. Pediatr Radiol 16:289-292

3. Sarwar A, Boland G, Monks A et al (2015) Metrics for radiologists in the era of value-based health care delivery. Radiographics 35:866876 